OPEN ACCESS

Edited by:

Kelong Ai,

Central South University, China

Reviewed by:

Zhen Liu,

Beijing University of Chemical Technology, China Yunlu Dai,

University of Macau, China

*Correspondence: Tingting Gong gongtt01@jlu.edu.cn Qinghai Yuan yqh@jlu.edu.cn

Specialty section: This article was submitted to

Pharmacology of Anti-Cancer Drugs,

a section of the journa

Frontiers in Oncology

Received: 14 July 2021 Accepted: 03 August 2021 Published: 26 August 2021

Citation:

Zhang $T$, Deng $M$, Zhang $L$, Liu Z, Liu Y, Song S, Gong T and Yuan Q (2021) Facile Synthesis

of Holmium-Based Nanoparticles as a CT and MRI Dual-Modal Imaging for Cancer Diagnosis.

Front. Oncol. 11:741383. doi: 10.3389/fonc.2021.741383

\section{Facile Synthesis of Holmium-Based Nanoparticles as a CT and MRI Dual- Modal Imaging for Cancer Diagnosis}

\author{
Tianqi Zhang ${ }^{1}$, Mo Deng ${ }^{2}$, Lei Zhang ${ }^{3}$, Zerun Liu ${ }^{4}$, Yang Liu ${ }^{5}$, Shuyan Song ${ }^{5}$, \\ Tingting Gong ${ }^{1 *}$ and Qinghai Yuan ${ }^{1 *}$ \\ ${ }^{1}$ Department of Radiology, The Second Hospital of Jilin University, Changchun, China, ${ }^{2}$ Department of Clinical Laboratory, \\ The Second Hospital of Jilin University, Changchun, China, ${ }^{3}$ Department of Neurology, The Second Hospital of Jilin \\ University, Changchun, China, ${ }^{4}$ Department of Clinical Pharmacy, Jilin University School of Pharmaceutical Science, \\ Changchun, China, ${ }^{5}$ State Key Laboratory of Rare Earth Resource Utilization, Changchun Institute of Applied Chemistry, \\ Chinese Academy of Sciences, Changchun, China
}

The rapid development of medical imaging has boosted the abilities of modern medicine. As single modality imaging limits complex cancer diagnostics, dual-modal imaging has come into the spotlight in clinical settings. The rare earth element Holmium (Ho) has intrinsic paramagnetism and great $\mathrm{X}$-ray attenuation due to its high atomic number. These features endow Ho with good potential to be a nanoprobe in combined $\mathrm{x}$-ray computed tomography $(\mathrm{CT})$ and $\mathrm{T}_{2}$-weighted magnetic resonance imaging (MRI). Herein, we present a facile strategy for preparing $\mathrm{HoF}_{3}$ nanoparticles $\left(\mathrm{HoF}_{3} \mathrm{NPs}\right)$ with modification by PEG 4000. The functional PEG-HoF $\mathrm{HPs}_{3}$ have good water solubility, low cytotoxicity, and biocompatibility as a dual-modal contrast agent. Currently, there is limited systematic and intensive investigation of $\mathrm{Ho}$-based nanomaterials for dual-modal imaging. Our PEG-HoF NPs provide a new direction to realize in vitro and vivo CT/MRI imaging, as well as validation of Ho-based nanomaterials will verify their potential for biomedical applications.

Keywords: nanomaterial, cancer, diagnosis, dual-model imaging, contrast agent

\section{INTRODUCTION}

Imaging technology plays an important role in modern medicine due to its ability to provide noninvasive but detailed information of anatomical structure and functional activities during the progress of a disease. However, single-model imaging methods cannot always meet the criteria for diagnosing co2mplex diseases $(1,2)$; thus, multi-modal imaging has become the new direction for imaging technology development (3-6). There are two main means to achieve multi-modal imaging (1): endow one device with multiple imaging capabilities; or (2) construct multi-modal contrast agents (CAs) for diagnosis. Conflicts between various methods and high costs have limited the feasibility of the first method. Hence, researchers are focusing their efforts on developing multimodal CAs that can be widely used in magnetic resonance imaging (MRI), X-ray computed tomography (CT), as well as fluorescence imaging (FI) (7-13). Due to the penetrative limitation of FI in vivo, CT and MRI are more commonly applied for clinical diagnosis (14-17). CT is the most cost-effective examination and offers strong X-ray penetration, which has very high resolution for bone and calcification but poor resolution for soft tissue. Notably, MRI can remedy this 
shortcoming. Therefore, the combination of CT and MRI can significantly improve diagnostic ability (2, 18-24).

At present, CT and MRI examinations are used independently in clinical settings. Small iodinated molecule nanomaterials are routinely applied as CT CAs to assist in disease diagnosis. Due to the lack of X-ray absorption, a very large amount of such CAs for intravenous injection is needed to meet the contrast requirement, usually $80-150 \mathrm{ml}$ per adult for angiography $(25,26)$. Such a high dose is not only likely to cause allergy, but it can also lead to irreversible renal damage (27-29). Furthermore, as small molecular iodide is quickly excreted by the kidney, its short circulation lifetime can affect imaging quality for certain diseases. Similarly, MRI has its own limitations. Gdchelates are the most commonly used commercial MRI CAs in clinical settings. However, Gd-based CAs can accumulate in the central nervous system and lead to renal fibrosis (30). Another notable disadvantage of MRI is that the optimum magnetic field strength for current Gd-based CAs is less than $1.0 \mathrm{~T}$ (31), whereas the trend in clinical MRI is to use ultra-high magnetic fields of $7.0 \mathrm{~T}$ or even higher because of the better imaging quality (32). 7.0 T MRI has been available in the market since 2017, and ultra-high field strength MRI equipment will become mainstream in the future. As a result, several researchers have been focusing on developing new CAs to suit this powerful visualization tool. $\mathrm{Tb}, \mathrm{Ho}$, and Dy are the elements with the largest magnetic moments in the periodic table and can cause considerable transverse relaxation of hydrogen protons in free water. Thus, Tb, Ho, and Dy-based materials are the best choices for ultra-high field $\mathrm{T}_{2}$ CAs (33-36). Against the background of continuous pursuit of ultra-high field intensity MRI, research on MRI CAs based on large magnetic moment elements is likely to experience rapid growth in the near future. Thus, we developed a facile strategy to construct control-sized $\mathrm{PEGylated} \mathrm{HoF}_{3}$ nanoparticles ( $\mathrm{PEG}-\mathrm{HoF}_{3} \mathrm{NPs}$ ) as a dual-modal imaging CA. Scheme 1. illustrates the design of our study. We used a facile one-pot solvothermal approach to obtain Ho-based nanomaterial, which has a high $\mathrm{X}$-ray attenuation and large magnetic moment. PEG-HoF $\mathrm{H}_{3} \mathrm{NPs}$ offer great biocompatibility and low cytotoxicity due to the existence of poly(ethylene glycol) (PEG). As a result, these NPs could be used as CT/MRI dualmodal imaging CAs both in vitro and in vivo, confirming the potential of Ho-based nanomaterials for bioapplication research.

\section{MATERIALS AND METHODS}

\section{Chemicals}

$\mathrm{HoCl}_{3} \cdot 6 \mathrm{H}_{2} \mathrm{O}$, poly(ethylene glycol) (PEG, MW $\left.=4000 \mathrm{Da}\right)$ was obtained from Sigma-Aldrich. $\mathrm{NH}_{4} \mathrm{~F}$ and ethylene glycol (EG) were obtained from Beijing Chemicals. Dimethyl sulfoxide (DMSO) were obtained from Sigma-Aldrich. DMEM and FBS were purchased from Gibco.

\section{Preparation of PEG-HoF 3 NPs}

PEG-HoF $\mathrm{NPs}_{3}$ were fabricated via a one-pot solvothermal method. Firstly, $0.8 \mathrm{mmol} \mathrm{HoCl}_{3} \cdot 6 \mathrm{H}_{2} \mathrm{O}$ was dissolved in $5 \mathrm{~mL}$

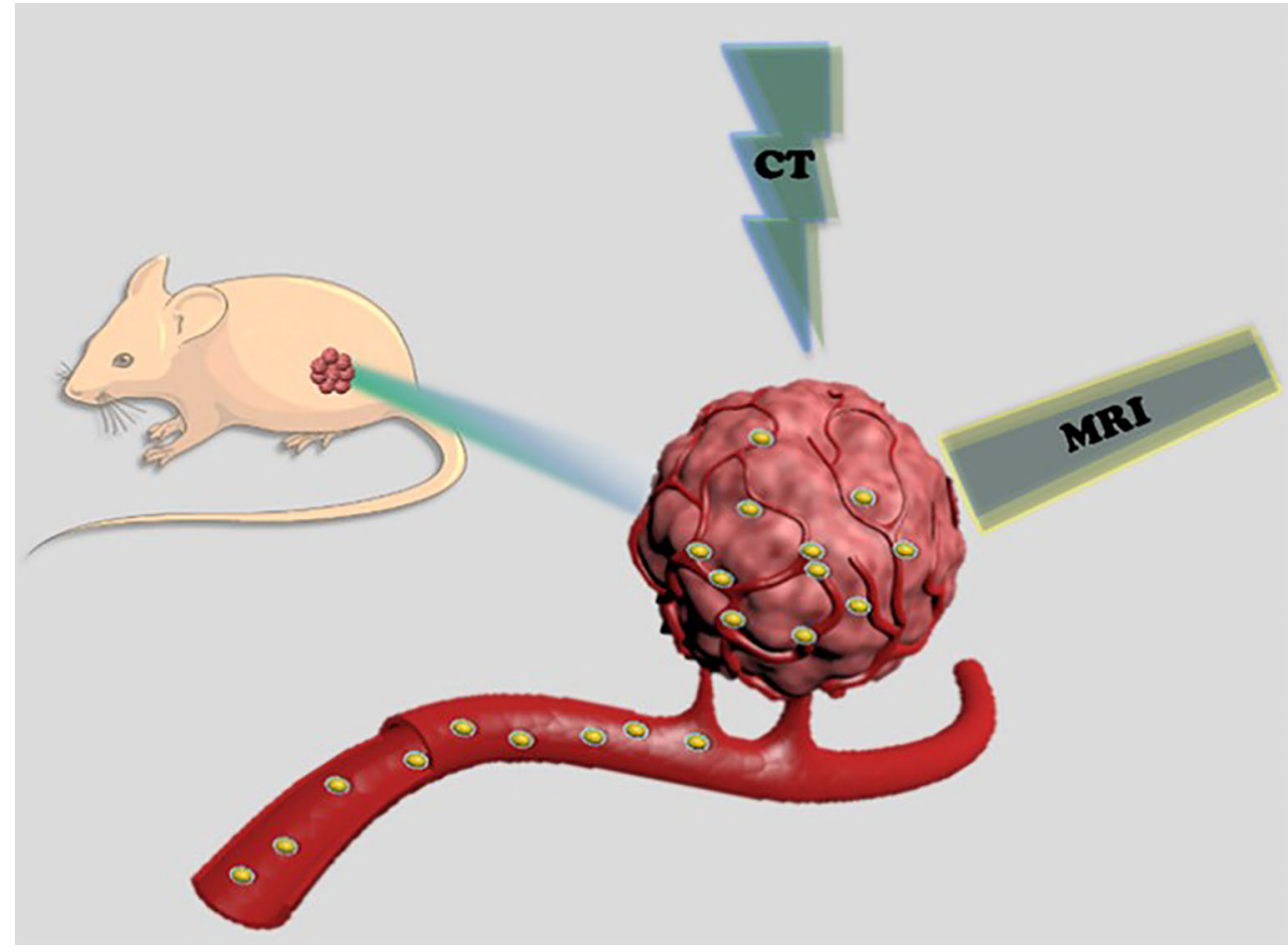

SCHEME 1 | Schematic illustration of PEG-HoF 3 NPs for enhanced CT and $\mathrm{T}_{2}$-weighted duel-model imaging. 
of EG to form a clear solution. Then, $0.6 \mathrm{~g}$ of PEG 4000 was added to $15 \mathrm{~mL}$ of EG to form a clear solution. Next, prepared EG with $2.4 \mathrm{mmol} \mathrm{NH}_{4} \mathrm{~F}$ added to the mixture of above two solutions. This mixed solutions were vigorously stirred for about $40 \mathrm{~min}$ and then transferred to a $50 \mathrm{~mL}$ Teflon-lined autoclave and kept at $200^{\circ} \mathrm{C}$ for $10 \mathrm{~h}$. When the system was cooled down, all collected NPs were washed several times.

\section{In Vitro Cytotoxicity Assessment}

Human cervix cancer cells (HeLa) and mouse fibroblast cells (L929) were cultured in DMEM with $10 \%$ FBS, $1 \%$ penicillin, and $1 \%$ streptomycin at a $37^{\circ} \mathrm{C}$ in a $5 \% \mathrm{CO}_{2}$ incubator. After incubation in 96-well cell culture plates for $24 \mathrm{~h}$, different concentrations of PEG-HoF $\mathrm{NPs}_{3}(0,25,50,100,200,300 \mu \mathrm{g} /$ $\mathrm{mL}$ ) were added to the HeLa cells and L929 cells and incubated for another $24 \mathrm{~h}$. Next, $10 \mu \mathrm{L}$ ( $5 \mathrm{mg} / \mathrm{mL}$ ) MTT was added to $96-$ well cell culture plates and kept for an additional $4 \mathrm{~h}$ at $37^{\circ} \mathrm{C}$. Finally, the medium was removed and DMSO was added for 15 min to dissolve the formazan. The absorbance peak at $570 \mathrm{~nm}$ was measured by a microplate reader.

\section{Histological and Hematology Assessment}

Kunming mice (18-25 g) were obtained from the Center for Experimental Animals, Jilin University (Changchun, China). All operations are carried out in accordance with relevant national regulations. For histological assessment, $100 \mu \mathrm{L}$ PEG-HoF $\mathrm{HPs}_{3}$ $(300 \mu \mathrm{g} / \mathrm{mL})$ and $100 \mu \mathrm{L} 0.9 \% \mathrm{NaCl}$ solution were administered to Kunming mice via tail vein. After 30 days, Major organs and tissues (heart, liver, spleen, lung, kidney, and muscle) were collected. All tissues samples were formalin-fixed, paraffinembedded, and stained with H\&E. Tissue sections were observed under an optical microscope $(\times 10)$. Blood samples were also obtained from the PEG- $\mathrm{HoF}_{3} \mathrm{NPs}$ and $\mathrm{NaCl}$ injected mouse groups for routine blood testing and biochemical indices testing.

\section{CT Imaging}

Different concentration of PEG- $\mathrm{HoF}_{3} \mathrm{NPs}$ and Iohexol aqueous solutions (I or Ho $0,0.25,0.5,1,2,4 \mathrm{mmol} / \mathrm{mL}$ ) were prepared for in vitro $\mathrm{CT}$ imaging. When the suitable con-centration was determined, in vivo CT images were obtained on Kunming mice. After intraperitoneal anesthesia with chloral hydrate, $100 \mu \mathrm{L}$ PEG- $\mathrm{HoF}_{3} \mathrm{NPs}(2 \mathrm{mg} / \mathrm{mL})$ were injected into these mice via tail vein and images were obtained at different times $(0,1,2,6,12,24$ h). CT was performed using a clinical CT scanner, and the parameters were tube voltage of $120 \mathrm{kVp}$, tube current of 300 mAs, $0.9 \mathrm{~mm}$ thickness, 0.99 pitch, window width of $200 \mathrm{HU}$, and window level of $45 \mathrm{HU}$.

\section{$\mathbf{T}_{2}$-Weighted MRI}

Different concentrations of PEG- $\mathrm{HoF}_{3} \mathrm{NPs}$ aqueous solutions (Ho $0,0.0625,0.125,0.25,0.5,1 \mathrm{mmol} / \mathrm{mL}$ ) were prepared for in vitro $\mathrm{T}_{2}$-weighted $\mathrm{MRI}$. Preliminary preparation was similar to the above steps. MRI was performed on a clinical MR scanner. $100 \mathrm{uL}$ PEG-HoF $\mathrm{H}_{3} \mathrm{NPs}(0.8 \mathrm{mg} / \mathrm{mL})$ were injected into mice as above. These mice were scanned pre-injection and 1,2, 4, 12, and
$24 \mathrm{~h}$ post-injection. The MR scanner parameters were $\mathrm{TR}=7279.7 \mathrm{~ms}, \mathrm{TE}=113 \mathrm{~ms}$ and $\mathrm{FOV}=240 \times 240 \mathrm{~mm}$.

\section{RESULTS AND DISCUSSION}

\section{Preparation and Characterization of PEG-HoF 3 NPs}

Uniform $\mathrm{HoF}_{3}$ NPs were prepared through a one-pot solvothermal method using PEG 4000 as a surfactant, as illustrated in Figure 1A. The TEM images demonstrate the good dispersivity of PEG- $\mathrm{HoF}_{3} \mathrm{NPs}$, and the existence of Ho and $\mathrm{F}$ element is proved by HADDF-STEM image and EDS mapping (Figures 1B-F), and the high-resolution TEM image shows that the width of lattice fringes about PEG-HoF $\mathrm{H}_{3} \mathrm{NPs}$ was $0.3401 \mathrm{~nm}$. The SEM image shows the spherical nanoparticles with an average diameter of $38 \mathrm{~nm}$; the diameter of PEG-HoF NPs was normally distributed in the range of 30-55 nm (Figure S1). In order to ascertain the hydrodynamic diameters of PEG-HoF $\mathrm{HPs}_{3}$, the dynamic light scattering (DLS) was performed, and the results show the average hydrodynamic diameter are suitable for using in organism in different solvents (Figure 2A). Meanwhile, the Zeta potential of PEG$\mathrm{HoF}_{3} \mathrm{NPs}$ was $5.83 \mathrm{mV}$ with the decoration of PEG. These value are suitable for nanomaterial which will be applied in vivo. XRD analysis exhibited several strong peaks, which indicated the highly crystalline nanostructure of this material. The diffraction peaks of the nanoparticles can be indexed to orthorhombic $\mathrm{HoF}_{3}$, which matches the card (PDF 00-0230284) in Figure 2B (34). XPS spectra verified that the NPs contained Ho 4d, F 1s (Figures 2C, D), further confirming the existence of $\mathrm{HoF}_{3}$ NPs. The peaks of $\mathrm{C}$ and $\mathrm{O}$ confirmed the presence of PEG (Figure S2). The peak in $3391 \mathrm{~cm}^{-1}$ of FTIR spectrum further confirm the existence of PEG (Figure S3). Due to the low toxicity and bio-tolerability of PEG, it was approved for use in biopharmaceuticals by the US FDA several years ago $(37,38)$. Using PEG 4000 to modify the surface of $\mathrm{HoF}_{3}$ NPs not only reduced cytotoxicity, but also enhanced dispersibility in water and phosphate-buffered saline (PBS) solution. The picture shows good stability of PEG- $\mathrm{HoF}_{3} \mathrm{NPs}$ in normal saline and PBS solution (Figure S4). There was no obvious $\mathrm{Ho}^{3+}$ ion dissociation after long-term dialysis in PBS solution $(\mathrm{pH}=7.4)$ with PEG-HoF 3 NPs.

\section{Biocompatibility Assessment of $\mathrm{PEG}^{-\mathrm{HoF}_{3} \text { NPs }}$}

Biocompatibility assessment should be carried out before nanomaterial imaging probes are applied in vivo. Due to the lack of data about the application of Ho-based nanomaterial in vivo, we carried out a variety of tests to assess the safety of PEG-HoF $\mathrm{NPs}_{3}$ The cytotoxicity of PEG-HoF $\mathrm{H}_{3} \mathrm{NP}$ was evaluated by the MTT cell proliferation assay. We chose HeLa and L929 cells to evaluate the toxicity of the new Ho-based nanomaterial by observing damage in cancer cells and normal cells, respectively. The cell viability results are depicted in Figures 3A, B. The two cell types were exposed to $\mathrm{PEG}-\mathrm{HoF}_{3} \mathrm{NPs}$ at different extracellular 

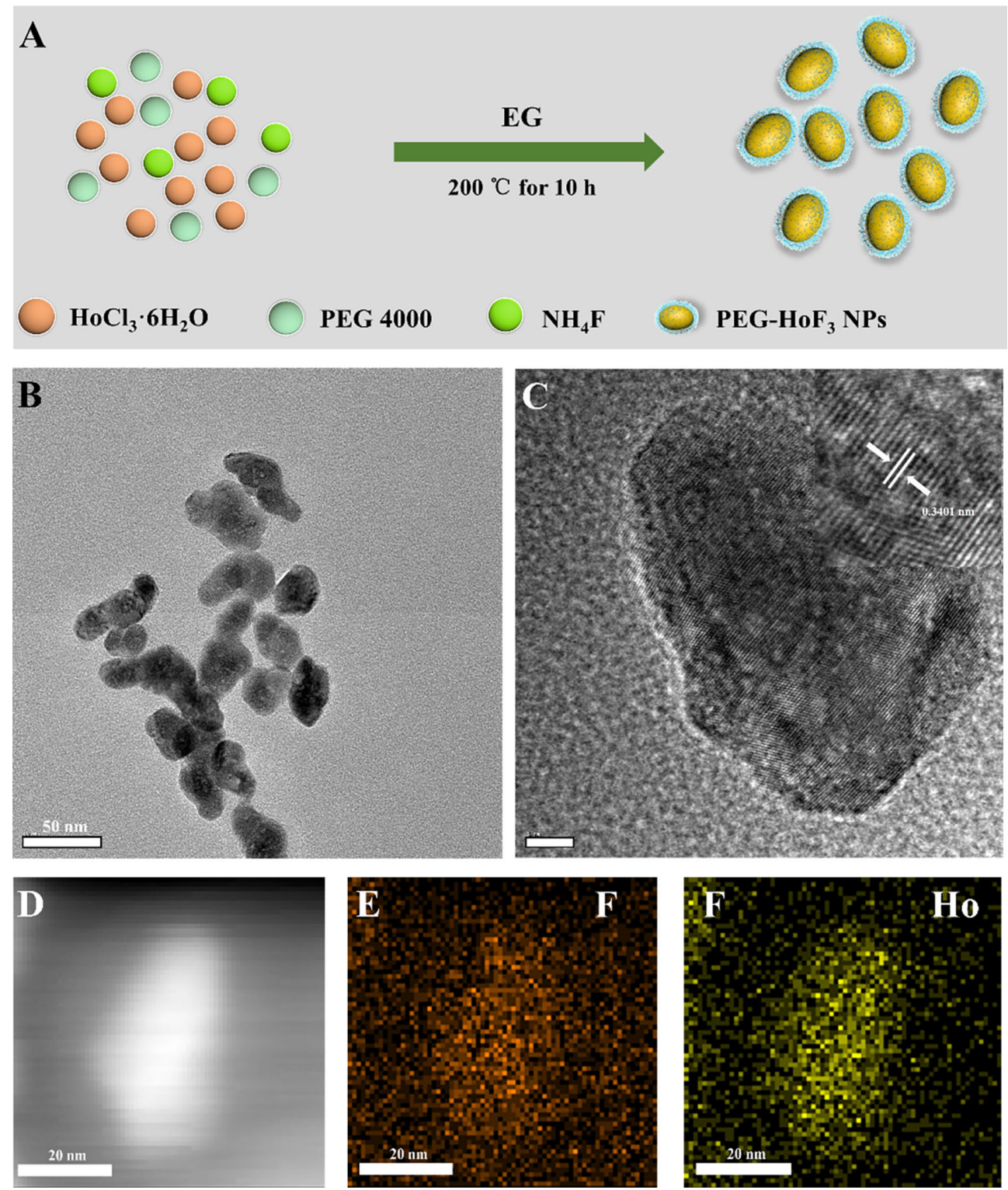

FIGURE 1 | (A) The synthetic process of PEG-HoF 3 NPs; (B, C) TEM image of PEG-HoF 3 NPs, the particles size is about 30-40nm and its lattice fringes; (D-F). HADDF-STEM image of PEG-HoF 3 and EDS mapping of $F$, Ho element.

concentrations $(0-300 \mu \mathrm{g} / \mathrm{mL})$. As expected, the new PEGylated Ho-based nanoparticles had great biocompatibility. Cell viability was still about $85 \%$ of the highest concentration $(300 \mu \mathrm{g} / \mathrm{mL})$ in both cell strains. We further investigated histological damage caused by PEG-HoF ${ }_{3}$ NPs through long-term toxicity assessment. Thirty days after injection, the two groups' mice were sacrificed. The main organs and tissues (heart, liver, spleen, lung, kidney, and muscle) underwent H\&E staining for histopathological assessment.

Paraffin sections conformed that there was no significant damage or severe inflammation in organs or tissues in either the control group or PEG-HoF $\mathrm{H}_{3} \mathrm{NPs}$ injection group (Figure 3C). Encouraged by these results, quantitative analysis of $\mathrm{PEG}-\mathrm{HoF}_{3} \mathrm{NPs}$ potential toxicity was carried out via blood testing and biochemical examination. Routine blood tests can be used to observe changes in the distribution of blood cells to judge the condition of mice, and biochemical examination can be used to detect various products of metabolism in blood to show the condition of organs such as liver or kidney. Thirty days after injection, there were no significant abnormalities in the morphology or indices of blood cells Figure 4. Metabolism of the main organs also remained stable, which was consistent with the physiological status of the mice. Taken together, the results confirmed that Ho-based nanomaterials offer great potential for bio-application researches due to their high biocompatibility. 

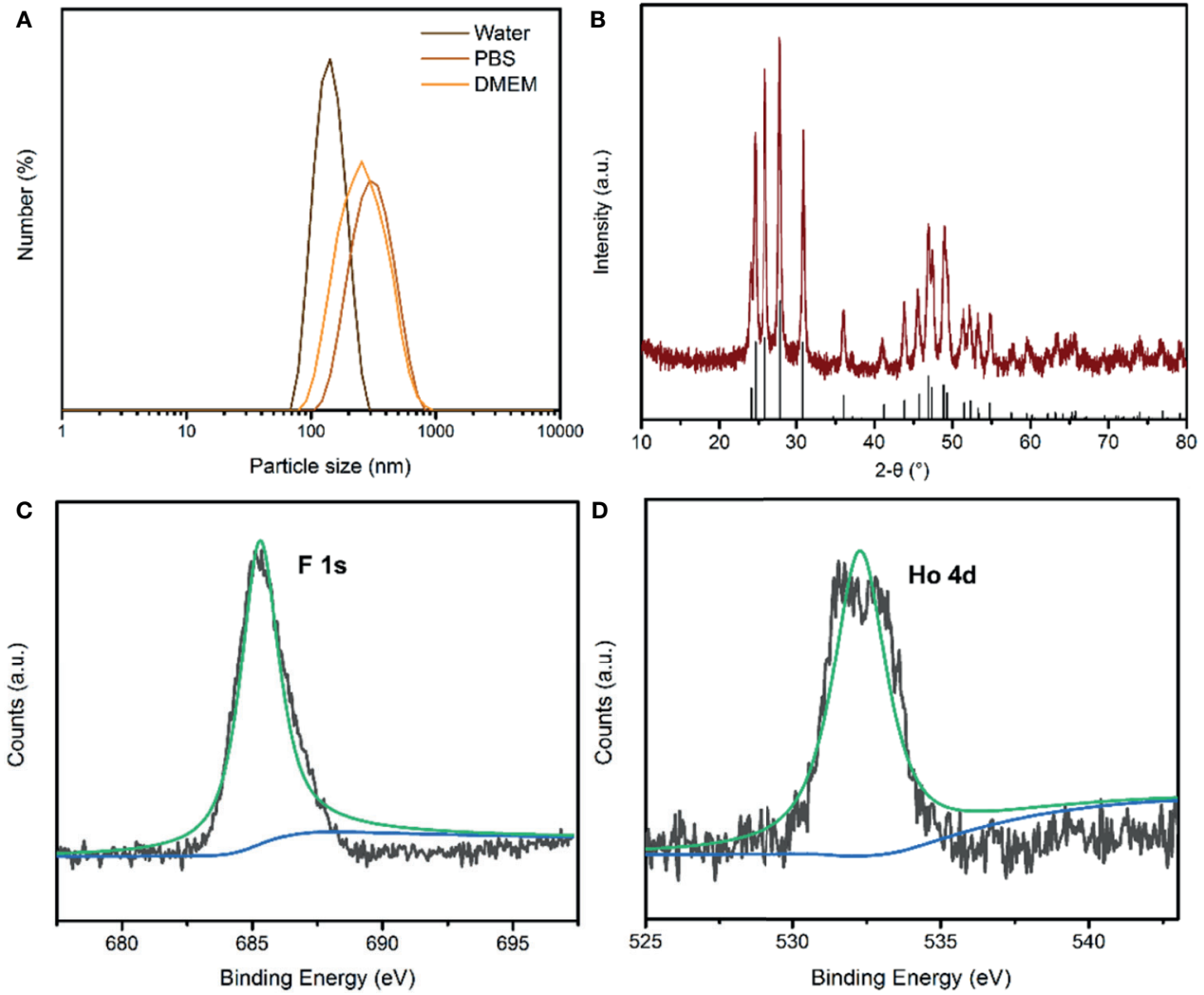

FIGURE 2 | (A) The dynamic light scattering (DLS) of PEG-HoF 3 NPs; (B) XRD pattern of PEG-HoF 3 NPs; (C, D) Higher-resolution XPS spectra of Ho 4d and F1s, respectively.
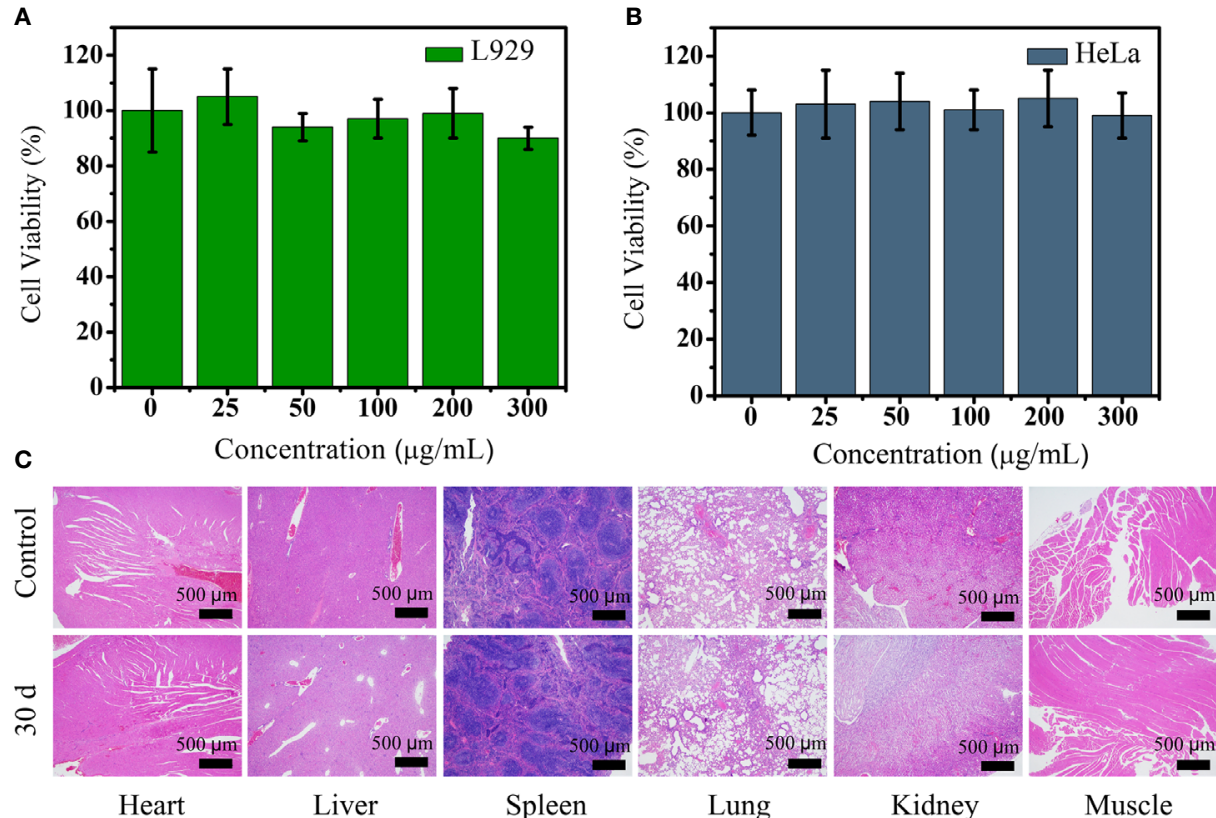

FIGURE 3 | (A, B) Viability of L929 and HeLa cells after incubation with different concentrations of PEG-HoF 3 NPs; (C) Tissue sections of 6 major organs from mouse which injected with $\mathrm{PEG}-\mathrm{HoF}_{3} \mathrm{NPs}$ via tail vein. 

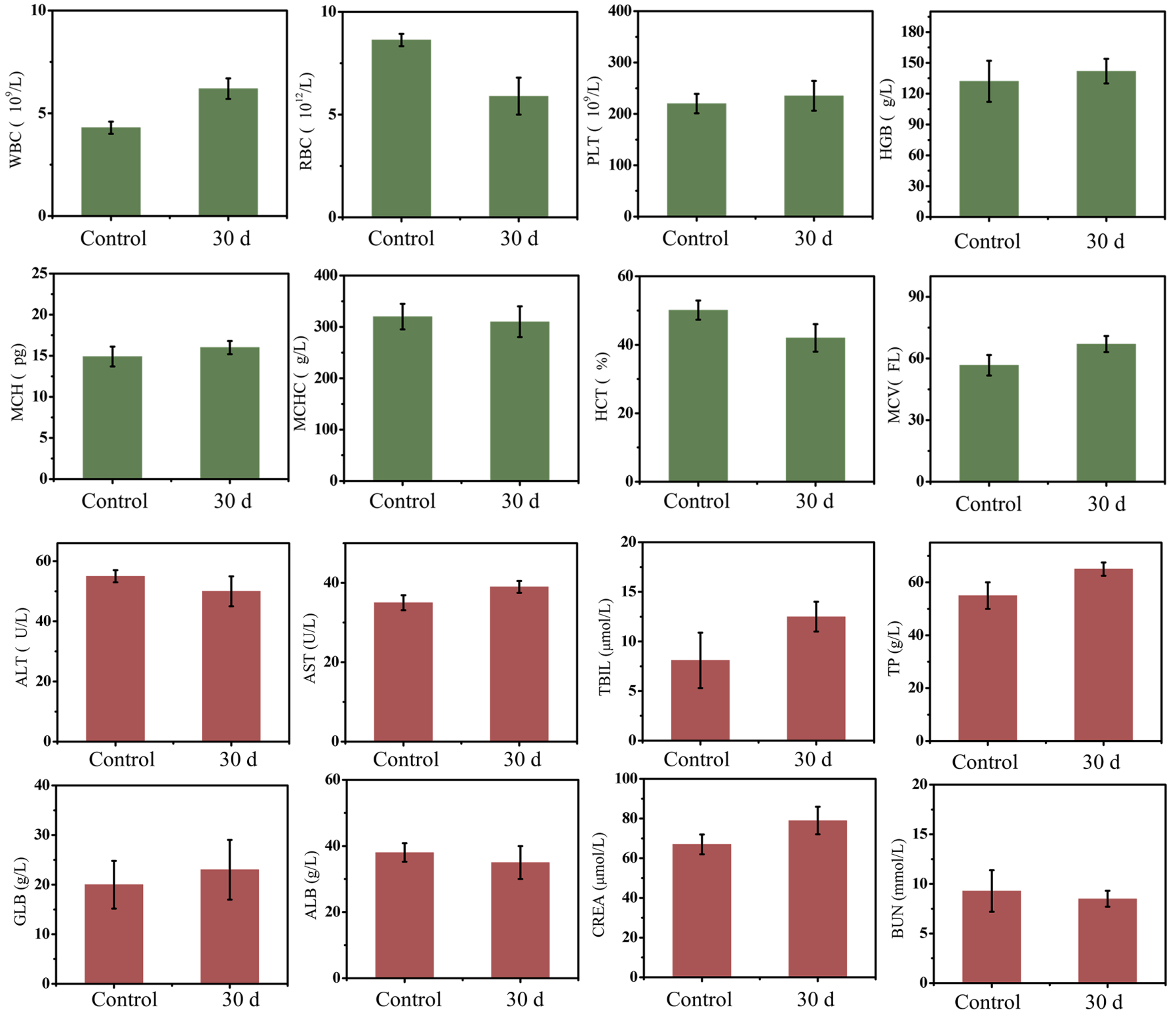

FIGURE 4 | Mice blood testing and biochemical examination, there is no evitable changes in two groups. 


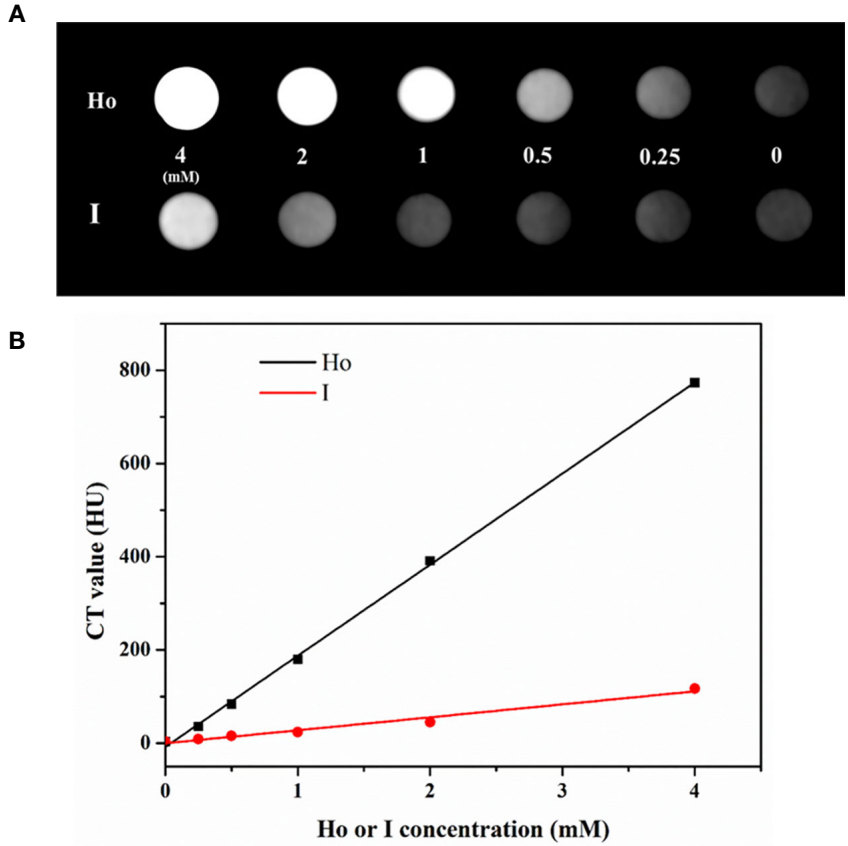

FIGURE 5 | (A) In vitro CT images of PEG-HoF ${ }_{3}$ NPs and lohexol aqueous solution with different concentration; (B) CT value of corresponding tube of A.

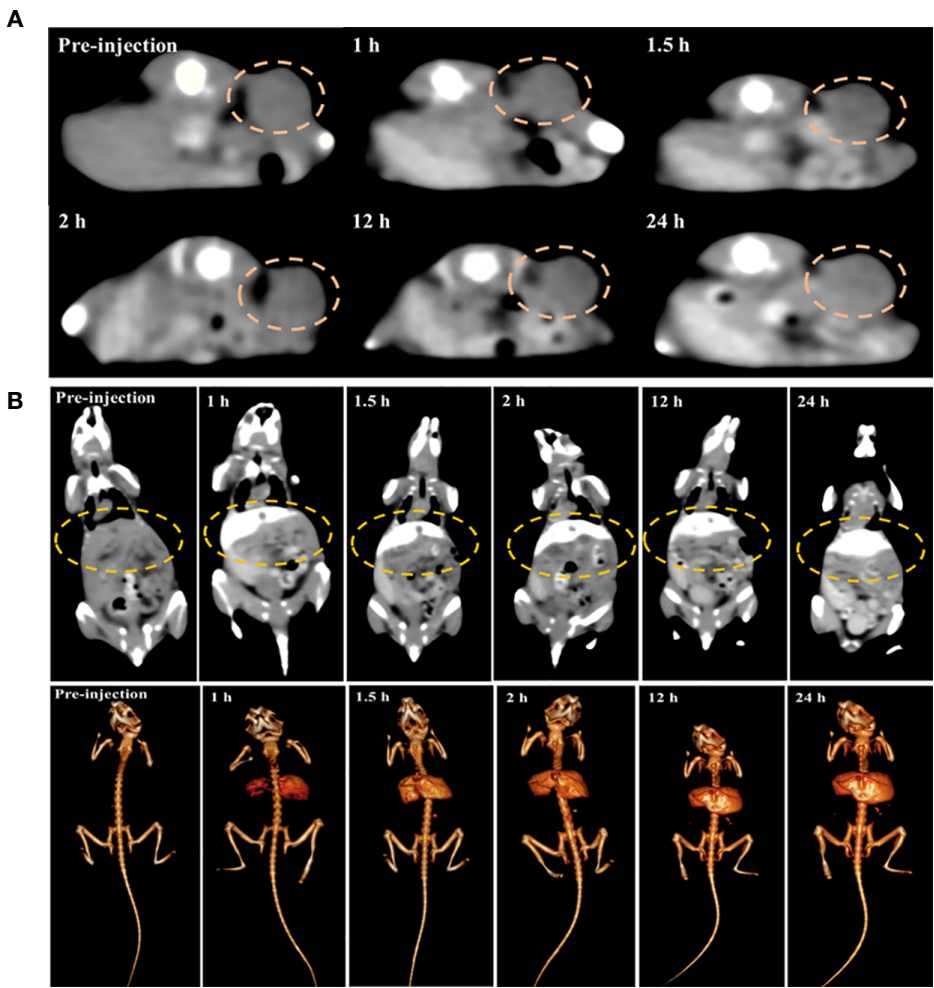

FIGURE 6 | (A, B) Changes of tumor site and mouse liver in different time point before versus post-injection of PEG-HoF NPs. 


\section{CT Imaging}

As a rare earth element, Ho has an atomic number of 67, which is much higher than iodine's atomic number of 53. The inherent characteristics of Ho, specifically its high $\mathrm{x}$-ray attenuation, result in the significantly improved imaging ability of Ho-based CAs in CT. The in vitro CT imaging results are shown in Figure 5A. Different concentrations of PEG-HoF $\mathrm{NPs}_{3}$ and Iohexol aqueous solution (0-4 $\mathrm{mM}$ ) were prepared in Eppendorf tubes and the solutions became brighter as the concentration increased. As shown in Figure 5B, the Hounsfield Units (HU) value of PEG$\mathrm{HoF}_{3}$ NPs was significantly higher than that of iodine-based CAs, as we expected, and the HU values for both increased linearly. Therefore, the necessary dose of $\mathrm{PEG}-\mathrm{HoF}_{3} \mathrm{NPs}$ is much lower than that of iodine-based CAs to achieve the same contrast effect in vivo, which may reduce the risks of large doses of CAs (39).

After cytotoxicity assessment of PEG- $\mathrm{HoF}_{3}$ NPs, tumorbearing Kunming mice were selected to test the CT contrast effect in vivo. The distribution of PEG- $\mathrm{HoF}_{3} \mathrm{NPs}$ was monitored by a CT scanner at different time points. We then compared the images at these time points with the images pre-injection: the brightness of the tumor site was obviously higher after $24 \mathrm{~h}$ (Figure 6A).

We also tracked the brightness of the liver and kidney after administering PEG-HoF $\mathrm{HP}_{3}$ NPs via the tail vein (Figures 6B and S5). Usually, small molecule iodine CAs are not long-lasting in the liver, which decreases the diagnostic ability of liver disease to some extent. However, as shown in Figure S5, the liver of the

A

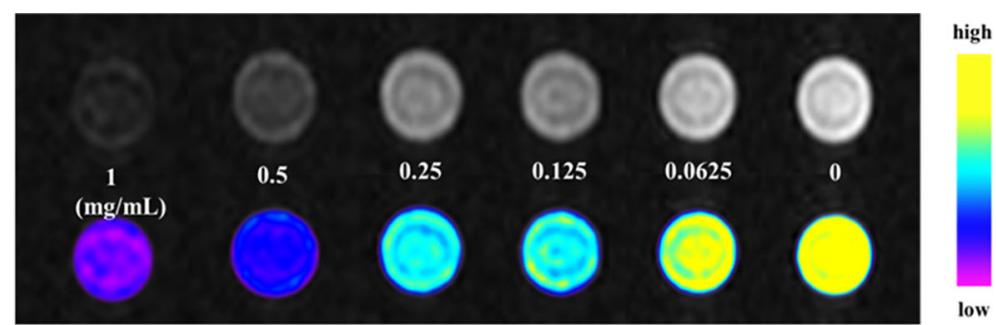

B

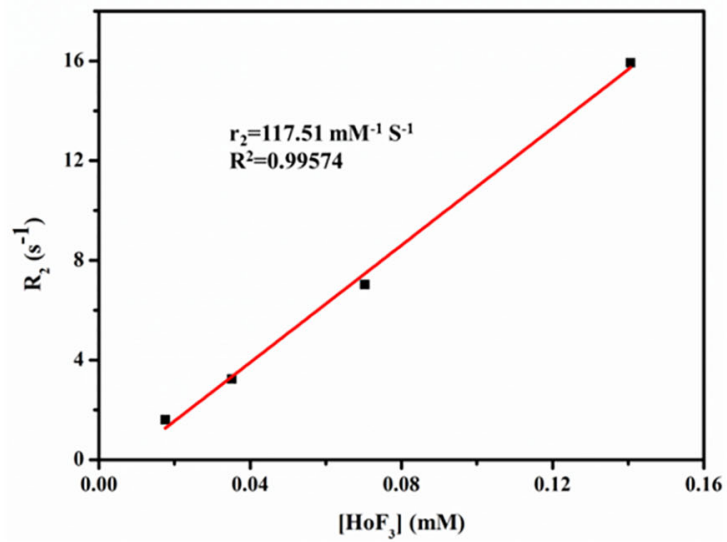

C
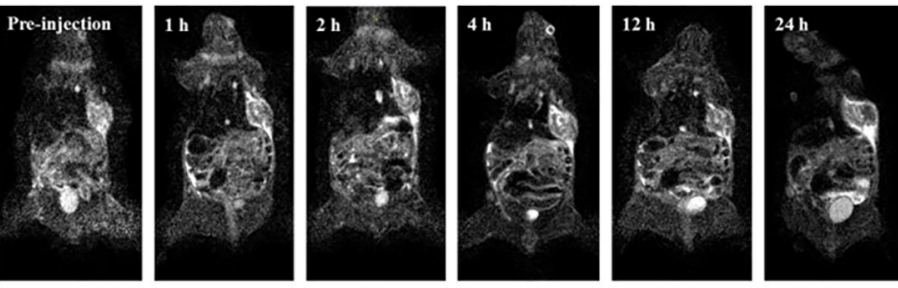

high
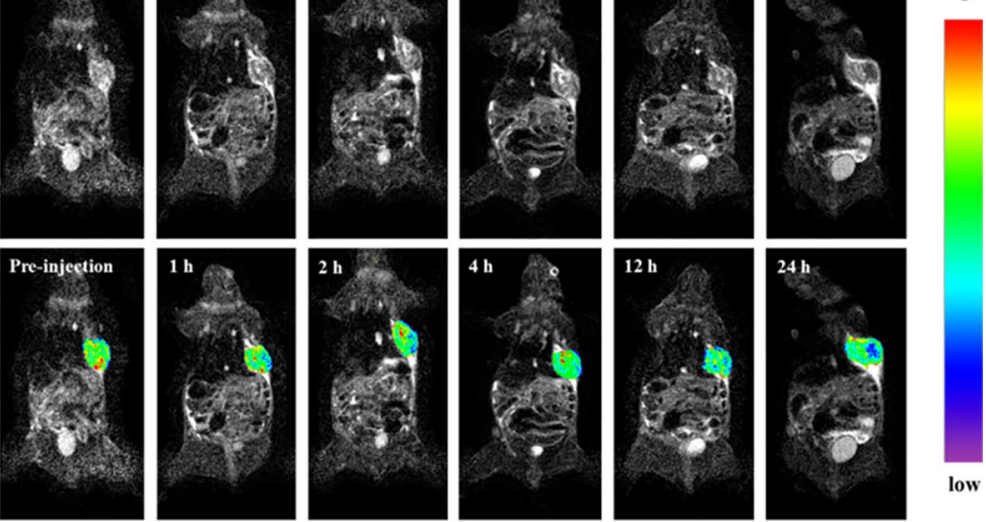

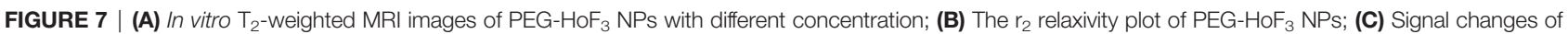
tumor site in different time point before and post-injection of PEG-HoF 3 NPs. 
mice maintained a high contrast effect after $24 \mathrm{~h}$, which likely indicates the uptake of PEG- $\mathrm{HoF}_{3} \mathrm{NPs}$ by hepatocytes. However, there was no obvious enhancement in the kidney at the time points we used. This may indicate that the uptake of PEG-HoF NPs by renal cells is limited and that the size and shape of PEG$\mathrm{HoF}_{3} \mathrm{NPs}$ prevented its filtration through the kidney. It is possible that the above mechanism can decrease the side effects of CAs on the kidney, and warrants further study.

\section{$\mathbf{T}_{2}$-Weighted MRI}

To date, there have been few studies on Ho-based MRI imaging. It has been confirmed that Ho-based nanomaterial has $\mathrm{T}_{2}$-weighted MRI contrast ability owing to its intrinsic paramagnetism. However, the magnetic resonance relaxivity of NPs can differ due to their size, form, and even synthesis methods. For this reason, we firstly validated the feasibility of $\mathrm{PEG}-\mathrm{HoF}_{3} \mathrm{NPs}$ as a $\mathrm{T}_{2}$-weighted MRI CAs in vitro. Different concentrations of $\mathrm{PEG}-\mathrm{HoF}_{3} \mathrm{NPs}$ aqueous solution were tested on a clinical MR scanner. $\mathrm{T}_{2}$-weighted images of PEG-HoF $\mathrm{HPs}_{3}$ aqueous solution $(0-1 \mathrm{mg} / \mathrm{mL})$ gradually darkened with an increasing Ho concentration (Figure 7A). According to the concentration of PEG-HoF $\mathrm{NPs}_{3}$, the $\mathrm{r}_{2}$ relaxivity value of this new contrast agent is $117.51 \mathrm{mM}^{-1} \mathrm{~S}^{-1}$ (Figure 7B). Next, we further explored the potential of PEG$\mathrm{HoF}_{3}$ NPs as negative MRI CAs in tumor-bearing mice. $\mathrm{T}_{2^{-}}$ weighted MRI was performed on the same scanner at different times $(1,2,4,12,24 \mathrm{~h})$ after intravenous injection of PEG-HoF $\mathrm{NPs}_{3}$ (Figure 7C). The results showed no significant changes in the signal of the tumor site within 0 to $2 \mathrm{~h}$. However, after $12 \mathrm{~h}$, the signal of the tumor site decreased significantly and a large dark area gradually presented. At $24 \mathrm{~h}$, the negative contrast agent filled the center of tumor site. These results also confirm long term circulation of PEG$\mathrm{HoF}_{3} \mathrm{NPs}$, which will be helpful for improving the detection of tumors at specific places.

\section{CONCLUSIONS}

We firstly synthesized a new Ho-based NP via a one-pot solvothermal method. The new PEG- $\mathrm{HoF}_{3} \mathrm{NPs}$ had a uniform size and showed good dispersibility in aqueous solution. Cytotoxicity assessment and histological analysis indicated that the new Ho-based NPs had good biocompatibility and low toxicity for applications in vivo. We then further verified the imaging ability of the PEG- $\mathrm{HoF}_{3} \mathrm{NPs}$ in vitro and vivo. The

\section{REFERENCES}

1. Shi J, Sun X, Zheng S, Li J, Fu X, Zhang H. A New Near-Infrared Persistent Luminescence Nanoparticle as a Multifunctional Nanoplatform for Multimodal Imaging and Cancer Therapy. Biomaterials (2018) 152:15-23. doi: 10.1016/j.biomaterials.2017.10.032

2. Padmanabhan P, Kumar A, Kumar S, Chaudhary RK, Gulyas B. Nanoparticles in Practice for Molecular-Imaging Applications: An Overview. Acta Biomater (2016) 41:1-16. doi: 10.1016/j.actbio.2016.06.003

3. Chen C, Liu J, Chen Y, Li C, Liu X, Huang H, et al. Sub-10 Nm Sr2LuF7:Yb/Er@ Sr2GdF7@SrF2 Up-Conversion Nanocrystals for Up-Conversion LuminescenceMagnetic Resonance-Computed Tomography Trimodal Bioimaging. ACS Appl Mater Interfaces (2017) 9(7):5748-56. doi: 10.1021/acsami.6b14007 results showed that $\mathrm{PEG}-\mathrm{HoF}_{3} \mathrm{NPs}$ had an excellent contrast effect in both CT and $\mathrm{T}_{2}$-weighted MRI. Based on this desirable performance, we expect that $\mathrm{PEG}-\mathrm{HoF}_{3} \mathrm{NPs}$ hold great promise for dual-modal imaging and that Ho-based nanomaterials warrant further research.

\section{DATA AVAILABILITY STATEMENT}

The original contributions presented in the study are included in the article/Supplementary Material. Further inquiries can be directed to the corresponding authors.

\section{ETHICS STATEMENT}

The animal study was reviewed and approved by Laboratory Animal Center of Jilin University.

\section{AUTHOR CONTRIBUTIONS}

TZ contributed to conception and design of the study. MD and $\mathrm{LZ}$ provided the database of mice. ZL contributed to the data of revised manuscript. YL and SS provided the testing instrument and site. TZ wrote the first draft of the manuscript. TG and QY revised and edited the manuscript. All authors contributed to the article and approved the submitted version.

\section{FUNDING}

This work was supported by the Program of Science and Technology Development Plan of Jilin Province of China (Nos. 20190201218JC), the Health Special Project of Jilin Province Department of Finance (Nos. 2019SCE7025, 2020SCZT088).

\section{SUPPLEMENTARY MATERIAL}

The Supplementary Material for this article can be found online at: https://www.frontiersin.org/articles/10.3389/fonc.2021. 741383/full\#supplementary-material.

4. Jin L, Liu J, Tang Y, Cao L, Zhang T, Yuan Q, et al. MnO2-Functionalized Co-P Nanocomposite: A New Theranostic Agent for $\mathrm{pH}$-Triggered T1/T2 Dual-Modality Magnetic Resonance Imaging-Guided Chemo-Photothermal Synergistic Therapy. ACS Appl Mater Interfaces (2017) 9(48):41648-58. doi: 10.1021/acsami.7b10608

5. Li Q, Li X, Zhang L, Zuo J, Zhang Y, Liu X, et al. An 800 Nm Driven NaErF4@ NaLuF4 Upconversion Platform for Multimodality Imaging and Photodynamic Therapy. Nanoscale (2018) 10(26):12356-63. doi: 10.1039/c8nr00446c

6. Ma S, Zhang J, Xia S, Yin W, Qin Y, Lei R, et al. Three-Dimensional Angiography Fused With CT/MRI for Multimodal Imaging of Nanoparticles Based on Ba4Yb3F17:Lu(3+),Gd(3+). Nanoscale (2018) 10 (28):13402-9. doi: 10.1039/c8nr03054e

7. van Schooneveld MM, Cormode DP, Koole R, van Wijngaarden JT, Calcagno C, Skajaa T, et al. A Fluorescent, Paramagnetic and PEGylated Gold/Silica 
Nanoparticle for MRI, CT and Fluorescence Imaging. Contrast Media Mol Imaging (2010) 5(4):231-6. doi: 10.1002/cmmi.376

8. Curcio A, Silva AKA, Cabana S, Espinosa A, Baptiste B, Menguy N, et al. Iron Oxide Nanoflowers @ CuS Hybrids for Cancer Tri-Therapy: Interplay of Photothermal Therapy, Magnetic Hyperthermia and Photodynamic Therapy. Theranostics (2019) 9(5):1288-302. doi: 10.7150/thno.30238

9. Zhang X, Xi ZQ, Machuki JO, Luo JJ, Yang DZ, Li JJ, et al. Gold Cube-In-Cube Based Oxygen Nanogenerator: A Theranostic Nanoplatform for Modulating Tumor Microenvironment for Precise Chemo-Phototherapy and Multimodal Imaging. ACS Nano (2019) 13(5):5306-25. doi: 10.1021/acsnano.8b09786

10. Wang ZX, Wu LM, Chen M, Zhou SX. Facile Synthesis of Superparamagnetic Fluorescent Fe3O4/ZnS Hollow Nanospheres. J Am Chem Soc (2009) 131 (32):11276-+. doi: 10.1021/ja903246e

11. Jeffrey RB. Imaging Pancreatic Cysts With CT and MRI. Digest Dis Sci (2017) 62(7):1787-95. doi: 10.1007/s10620-017-4501-6

12. Schieda N, Lim RS, McInnes MDF, Thomassin I, Renard-Penna R, Tavolaro S, et al. Characterization of Small $(<4 \mathrm{~cm})$ Solid Renal Masses by Computed Tomography and Magnetic Resonance Imaging: Current Evidence and Further Development. Diagn Intervent Imaging (2018) 99(7-8):443-55. doi: 10.1016/j.diii.2018.03.004

13. Ahlawat S, Baig A, Blakeley JO, Jacobs MA, Fayad LM. Multiparametric Whole-Body Anatomic, Functional, and Metabolic Imaging Characteristics of Peripheral Lesions in Patients With Schwannomatosis. J Magnet Resonance Imaging: JMRI (2016) 44(4):794-803. doi: 10.1002/jmri.25236

14. Shan X, Chen Q, Yin X, Jiang C, Li T, Wei S, et al. Polypyrrole-Based Double Rare Earth Hybrid Nanoparticles for Multimodal Imaging and Photothermal Therapy. J Mater Chem B (2020) 8(3):426-37. doi: 10.1039/c9tb02254f

15. Lyu M, Zhu DM, Duo YH, Li Y, Quan H. Bimetallic Nanodots for Tri-Modal CT/MRI/PA Imaging and Hypoxia-Resistant Thermoradiotherapy in the NIR-II Biological Windows. Biomaterials (2020) 233:119656. doi: 10.1016/ j.biomaterials.2019.119656

16. Li Z, Guan M, Sun D, Xu Y, Li F, Xiong W. A Novel MRI- and CT-Based Scoring System to Differentiate Malignant From Osteoporotic Vertebral Fractures in Chinese Patients. BMC Musculoskelet Disord (2018) 19(1):406. doi: 10.1186/s12891-018-2331-0

17. Jin X, Fang F, Liu J, Jiang C, Han X, Song Z, et al. An Ultrasmall and Metabolizable PEGylated NaGdF4:Dy Nanoprobe for High-Performance T (1)/T(2)-Weighted MR and CT Multimodal Imaging. Nanoscale (2015) 7 (38):15680-8. doi: 10.1039/c5nr04065e

18. Park EA, Lee W, Kang DK, Kim SJ, Kim YJ, Kim Y, et al. Comparison of Iohexol-380 and Iohexol-350 for Coronary CT Angiography: A Multicenter, Randomized, Double-Blind Phase 3 Trial. Korean J Radiol (2016) 17(3):330-8. doi: 10.3348/kjr.2016.17.3.330

19. Bolen MA, Brinza E, Renapurkar RD, Kim ESH, Gornik HL. Screening CT Angiography of the Aorta, Visceral Branch Vessels, and Pelvic Arteries in Fibromuscular Dysplasia. JACC Cardiovasc Imaging (2017) 10(5):554-61. doi: 10.1016/j.jcmg.2016.04.010

20. Nijssen EC, Rennenberg RJ, Nelemans PJ, Essers BA, Janssen MM, Vermeeren MA, et al. Prophylactic Hydration to Protect Renal Function From Intravascular Iodinated Contrast Material in Patients at High Risk of Contrast-Induced Nephropathy (AMACING): A Prospective, Randomised, Phase 3, Controlled, Open-Label, Non-Inferiority Trial. Lancet (2017) 389 (10076):1312-22. doi: 10.1016/S0140-6736(17)30057-0

21. Wang Z, Jia T, Sun QQ, Kuang Y, Liu B, Xu MS, et al. Construction of Bi/ phthalocyanine Manganese Nanocomposite for Trimodal Imaging Directed Photodynamic and Photothermal Therapy Mediated by $808 \mathrm{Nm}$ Light. Biomaterials (2020) 228:119569. doi: 10.1016/j.biomaterials.2019.119569

22. Ai K, Huang J, Xiao Z, Yang Y, Bai Y, Peng J. Localized Surface Plasmon Resonance Properties and Biomedical Applications of Copper Selenide Nanomaterials. Mater Today Chem (2021) 20:100402. doi: 10.1016/j.mtchem.2020.100402

23. Wang J, Sui L, Huang J, Miao L, Nie Y, Wang K, et al. MoS2-Based Nanocomposites for Cancer Diagnosis and Therapy. Bioactive Mater (2021) 6(11):4209-42. doi: 10.1016/j.bioactmat.2021.04.021

24. Huang Q, Wu W, Ai K, Liu J. Highly Sensitive Polydiacetylene Ensembles for Biosensing and Bioimaging. Front Chem (2020) 8:565782. doi: 10.3389/ fchem.2020.565782

25. Estelrich J, Sanchez-Martin MJ, Busquets MA. Nanoparticles in Magnetic Resonance Imaging: From Simple to Dual Contrast Agents. Int J Nanomed (2015) 10:1727-41. doi: 10.2147/IJN.S76501
26. Rohrer M, Bauer H, Mintorovitch J, Requardt M, Weinmann HJ. Comparison of Magnetic Properties of MRI Contrast Media Solutions at Different Magnetic Field Strengths. Invest Radiol (2005) 40(11):715-24. doi: 10.1097/ 01.rli.0000184756.66360.d3

27. Kraff O, Quick HH. 7t: Physics, Safety, and Potential Clinical Applications. J Magnet Resonance Imaging: JMRI (2017) 46(6):1573-89. doi: 10.1002/jmri.25723

28. Chen L, Huang Q, Zhao T, Sui L, Wang S, Xiao Z, et al. Nanotherapies for Sepsis by Regulating Inflammatory Signals and Reactive Oxygen and Nitrogen Species: New Insight for Treating COVID-19. Redox Biol (2021) 45:102046. doi: 10.1016/j.redox.2021.102046

29. Sui L, Wang J, Xiao Z, Yang Y, Yang Z, Ai K. ROS-Scavenging Nanomaterials to Treat Periodontitis. Front Chem (2020) 8:595530. doi: 10.3389/ fchem.2020.595530

30. Ni D, Zhang J, Bu W, Zhang C, Yao Z, Xing H, et al. PEGylated NaHoF4 Nanoparticles as Contrast Agents for Both X-Ray Computed Tomography and Ultra-High Field Magnetic Resonance Imaging. Biomaterials (2016) 76:218-25. doi: 10.1016/j.biomaterials.2015.10.063

31. Gonzalez-Mancebo D, Becerro AI, Rojas TC, Garcia-Martin ML, de la Fuente JM, Ocana M. HoF3 and DyF3 Nanoparticles as Contrast Agents for HighField Magnetic Resonance Imaging. Part Part Syst Char (2017) 34(10): 1700116. doi: 10.1002/Ppsc.201700116

32. Das GK, Johnson NJ, Cramen J, Blasiak B, Latta P, Tomanek B, et al. NaDyF4 Nanoparticles as T2 Contrast Agents for Ultrahigh Field Magnetic Resonance Imaging. J Phys Chem Lett (2012) 3(4):524-9. doi: 10.1021/jz201664h

33. Marasini S, Yue H, Ho SL, Jung KH, Park JA, Cha H, et al. D-Glucuronic Acid-Coated Ultrasmall Paramagnetic $\operatorname{Ln}(2) \mathrm{O}(3)(\operatorname{Ln}=\mathrm{Tb}$, Dy, and $\mathrm{Ho})$ Nanoparticles: Magnetic Properties, Water Proton Relaxivities, and Fluorescence Properties. Eur J Inorg Chem (2019) 2019(34):3832-9. doi: 10.1002/ejic.201900378

34. D'Souza AA, Shegokar R. Polyethylene Glycol (PEG): A Versatile Polymer for Pharmaceutical Applications. Expert Opin Drug Deliv (2016) 13(9):1257-75. doi: 10.1080/17425247.2016.1182485

35. Ai K, Liu Y, Liu J, Yuan Q, He Y, Lu L. Large-Scale Synthesis of Bi(2)S(3) Nanodots as a Contrast Agent for In Vivo X-Ray Computed Tomography Imaging. Adv Mater (2011) 23(42):4886-91. doi: 10.1002/adma.201103289

36. Liu JH, Wang L, Zhang TQ, Wang JQ, Gong X, Cui FZ, et al. Facile Synthesis of Biocompatible Fe3O4-Based Nanoparticles for pH-Responsive Dual-Model Magnetic Resonance Imaging- Guided Tumour Eradication by Photothermal Therapy. Chin J Anal Chem (2019) 47(5):678-85. doi: 10.1016/S1872-2040 (19)61158-8

37. Zhang TQ, Han XL, He YY, He XJ, Wang JQ, Yuan QH, et al. Facile Synthesis of PEGylated Tungsten-Based Nanoprobes for Gastric Computed Tomography Imaging. Chin J Anal Chem (2018) 46(10):1539-43. doi: 10.1016/S1872-2040(18)61116-8

38. Li NS, Chen L, Xiao ZX, Yang YQ, Ai KL. Progress in Detection of Biomarker of Ovarian Cancer: Lysophosphatidic Acid. Chin J Anal Chem (2020) 48 (12):1597-606. doi: 10.19756/j.issn.0253.3820.201339

39. Zhu S, Tian R, Antaris AL, Chen X, Dai H. Near-Infrared-II Molecular Dyes for Cancer Imaging and Surgery. Adv Mater (2019) 31(24):e1900321. doi: 10.1002/adma.201900321

Conflict of Interest: The authors declare that the research was conducted in the absence of any commercial or financial relationships that could be construed as a potential conflict of interest.

Publisher's Note: All claims expressed in this article are solely those of the authors and do not necessarily represent those of their affiliated organizations, or those of the publisher, the editors and the reviewers. Any product that may be evaluated in this article, or claim that may be made by its manufacturer, is not guaranteed or endorsed by the publisher.

Copyright ( 2021 Zhang, Deng, Zhang, Liu, Liu, Song, Gong and Yuan. This is an open-access article distributed under the terms of the Creative Commons Attribution License (CC BY). The use, distribution or reproduction in other forums is permitted, provided the original author(s) and the copyright owner(s) are credited and that the original publication in this journal is cited, in accordance with accepted academic practice. No use, distribution or reproduction is permitted which does not comply with these terms. 